\title{
Isolation and characterization of equine native MSC populations
}

Cristina L. Esteves ${ }^{1,4^{*}}$, Tara A. Sheldrake ${ }^{1}$, Simone P. Mesquita', Juan J. Pesántez ${ }^{1}$, Timothy Menghini', Lucy Dawson', Bruno Péault²,3 and F. Xavier Donadeu'

\begin{abstract}
Background: In contrast to humans in which mesenchymal stem/stromal cell (MSC) therapies are still largely in the clinical trial phase, MSCs have been used therapeutically in horses for over 15 years, thus constituting a valuable preclinical model for humans. In human tissues, MSCs have been shown to originate from perivascular cells, namely pericytes and adventitial cells, which are identified by the presence of the cell surface markers CD146 and CD34, respectively. In contrast, the origin of MSCs in equine tissues has not been established, preventing the isolation and culture of defined cell populations in that species. Moreover, a comparison between perivascular CD146 ${ }^{+}$ and $\mathrm{CD} 34^{+}$cell populations has not been performed in any species.

Methods: Immunohistochemistry was used to identify adventitial cells $\left(\mathrm{CD} 34^{+}\right)$and pericytes $\left(\mathrm{CD} 146^{+}\right)$and to determine their localization in relation to MSCs in equine tissues. Isolation of $\mathrm{CD}_{4} 4^{+}$(CD34 ${ }^{+} / \mathrm{CD}_{146} / \mathrm{CD} 144^{-} / \mathrm{CD}_{4} 5^{-}$) and $\mathrm{CD}_{146^{+}}\left(\mathrm{CD} 146^{+} / \mathrm{CD} 34^{-} / \mathrm{CD} 144^{-} / \mathrm{CD}^{-} 5^{-}\right)$cell fractions from equine adipose tissue was achieved by fluorescenceactivated cell sorting. The isolated cell fractions were cultured and analyzed for the expression of MSC markers, using qPCR and flow cytometry, and for the ability to undergo trilineage differentiation. Angiogenic properties were analyzed in vivo using a chorioallantoic membrane (CAM) assay.
\end{abstract}

Results: Both $\mathrm{CD}_{4} 4^{+}$and $\mathrm{CD} 146^{+}$cells displayed typical MSC features, namely growth in uncoated tissue culture dishes, clonal growth when seeded at low density, expression of typical MSC markers, and multipotency shown by the capacity for trilineage differentiation. Of note, $\mathrm{CD}_{146}{ }^{+}$cells were distinctly angiogenic compared with $\mathrm{CD} 34^{+}$and non-sorted cells (conventional MSCS), demonstrated by the induction of blood vessels in a CAM assay, expression of elevated levels of VEGFA and ANGPT1, and association with vascular networks in cocultures with endothelial cells, indicating that $\mathrm{CD} 146^{+}$cells maintain a pericyte phenotype in culture.

Conclusion: This study reports for the first time the successful isolation and culture of CD146 ${ }^{+}$and $\mathrm{CD} 34^{+}$cell populations from equine tissues. Characterization of these cells evidenced their distinct properties and MSC-like phenotype, and identified $\mathrm{CD} 146^{+}$cells as distinctly angiogenic, which may provide a novel source for enhanced regenerative therapies.

Keywords: Pericyte, Equine, Horse, Adventitial cells, CD146, CD34, Adipose tissue

\footnotetext{
* Correspondence: cristina.esteves@roslin.ed.ac.uk

${ }^{1}$ The Roslin Institute, University of Edinburgh, Edinburgh, UK

${ }^{4}$ The Roslin Institute, University of Edinburgh, Easter Bush, Midlothian EH25

9RG, UK

Full list of author information is available at the end of the article
} 


\section{Background}

Other than humans, horses are the species in which interest in the use of mesenchymal stem/stromal cells (MSCs) for regenerative medicine has been highest [1-8]. Similar to humans, adipose tissue and bone marrow have been the MSC sources of choice in the horse, due to their relative ease of access and the number of putative stem cells they contain [2, 9-13]. Indeed, MSCs have been safely applied clinically to horses for well over a decade, now being an integral component of equine health care worldwide which has an estimated economic impact in the United States and the United Kingdom of $\$ 102$ billion and $\$ 10$ billion per annum, respectively. Importantly, given the similarities in the types of natural injuries suffered by both humans and horses, the US Food and Drug Administration [14] has approved the use of horses as a preclinical model for musculoskeletal conditions.

In regenerative medicine, cell preparations are applied either as crude extracts or following expansion in culture to attain the desired amount of clinical cells. However, these preparations are heterogeneous in nature, containing small and variable amounts of true stem cells in addition to other, nonprecursor cell types which upon transplantation into patients may reduce therapeutic efficacy. There is therefore a real need to identify specific subsets of cells that have intrinsic regenerative capacity, and to develop strategies to robustly isolate, expand in culture, and characterize these cells. However, there is a fundamental lack of understanding of the precise identity and defining features of MSCs in equine tissues, which has thwarted efforts to selectively and efficiently identify, harvest, and expand these cell subpopulations in culture.

Studies in humans have identified two distinct perivascular cell subpopulations, pericytes in capillaries and microvessels $[15,16]$ and adventitial cells in arteries and veins [17], as in vivo counterparts of MSCs [18, 19]. Pericytes and adventitial cells were isolated based on the presence of the cell surface markers CD146 and CD34, respectively, and were shown to maintain a typical MSC phenotype in culture. Although tissue regeneration potential has been demonstrated for each individual cell subpopulation [20-22], the properties of CD146 and CD34 cells have not been compared directly.

Using fluorescence-activated cell sorting (FACS), we report the isolation, culture, and characterization of equine $\mathrm{CD} 4^{+} / \mathrm{CD} 146^{-} / \mathrm{CD} 144^{-} / \mathrm{CD} 45^{-}$(adventitial cells) and CD $146^{+} / \mathrm{CD} 34^{-} / \mathrm{CD} 144^{-} / \mathrm{CD} 45^{-}$(pericytes) fractions-for simplicity, referred to hereafter as $\mathrm{CD} 34^{+}$and $\mathrm{CD} 146^{+}$, respectively. Both cell types displayed an MSC phenotype, although comparison with non-sorted cells (conventional cultured MSCs) revealed differences in expression of cell surface markers and angiogenic genes. Remarkably,
CD $146^{+}$cells were distinctly able to promote angiogenesis in vivo, highlighting their potential for tissue regeneration.

\section{Methods \\ Samples}

Tissue samples were obtained from a total of 15 adult horses immediately post mortem at the School of Veterinary Studies of the University of Edinburgh. All animal procedures were carried out according to the UK Home Office Animals (Scientific Procedures) Act 1986 with approval by the Ethical Review Committee, University of Edinburgh, 60/4207. Adipose tissue was kept for up to $24 \mathrm{~h}$ at $4{ }^{\circ} \mathrm{C}$ [23] before extraction of the stromal vascular fraction (SVF).

\section{Immunohistochemistry}

Frozen samples, processed immediately after tissue harvesting, were cut in a Leica CM1900 cryostat. For staining, tissues were fixed in ice-cold acetone:methanol (50:50) and antibodies were prepared in antibody diluent (003118; Invitrogen-Thermo Fisher Scientific, Paisley, UK). After staining, slides were mounted in Fluoroshield with 4',6-diamidino-2-phenylindole (DAPI;F6057; Sigma, St Louis, MO, USA). Primary antibodies used were CD146-FITC (MCA2141F; AbD Serotec-BioRad, Kidlington, UK), CD144 (AHP628Z; AbD Serotec-BioRad), NG2 (MAB2585; R\&D Systems, Minneapolis, MN, USA), aSMA (ab5694; AbCam, Cambridge, UK), CD29 (303015; BioLegend, San Diego, CA, USA), CD44 (AbD SerotecBioRad), and CD34 (21270341S; ImmunoTools, Friesoythe, Germany). Isotype controls were IgG1-FITC (MCA928F; AbD Serotec-BioRad), IgG1 (MAB002; R\&D Systems), IgG1к (400101; BioLegend), and IgG1 (21335011; ImmunoTools), all raised in mouse; and rabbit IgG (PRABP01; AbD Serotec-BioRad). Secondary antibodies were conjugated to AF488 (A11029), AF568 (A110037), and AF568 (A10042), all from InvitrogenThermo Fisher Scientific. Micrographs were produced using a Zeiss LSM710 confocal or Leica DMLB fluorescent microscope.

\section{Cell extraction and culture}

Extraction of the SVF from adipose tissue involved mincing the tissue with scissors and digestion for $45 \mathrm{~min}$ with collagenase II $(1 \mathrm{mg} / \mathrm{ml}$; Gibco-Thermo Fisher Scientific)/BSA (3.5\%) at $37{ }^{\circ} \mathrm{C}$ under agitation (100 rpm). Collagenase activity was stopped by addition of DMEM with 20\% FBS (Thermo Fisher Scientific) and the lipid layer was removed after separation by gravity. Samples were filtered and spun down to obtain the SVF, which was further washed in PBS/FBS (5\%) and treated with red cell lysis buffer (Sigma-Aldrich). Cells were then stained with appropriate antibodies for flow analysis or 
sorting, or seeded for expansion at $5000 \mathrm{cells} / \mathrm{cm}^{2}$ in EGM-2 (Lonza, Slough, UK) which was replaced by DMEM/FBS (20\%) at the first cell passage. The doubling time of non-sorted, $\mathrm{CD} 34^{+}$, or $\mathrm{CD} 146^{+}$cultured cells was calculated using the formula:

$$
\mathrm{DT}=T \ln 2 / \ln \left(X_{\mathrm{e}} / X_{\mathrm{b}}\right),
$$

where $T$ is the incubation time in days, $X_{\mathrm{b}}$ is the cell number at the beginning of the incubation time, and $X_{e}$ is the cell number at the end of the incubation time. To determine colony-forming unit fibroblasts (CFU-F), cells were seeded at a density of 1,5 , and 25 cells $/ \mathrm{cm}^{2}$ and allowed to grow for 12 days. Colonies were visualized by staining with crystal violet $(0.5 \%)$ and counted. To obtain endothelial cells, blood vessel sections were washed with PBS and then filled with collagenase II solution $(1 \mathrm{mg} / \mathrm{ml})$ before both ends of the vessel were ligated. Following 45-min incubation at $37{ }^{\circ} \mathrm{C}$, endothelial cells were recovered and were cultured in EGM-2 endothelial cell medium.

\section{Flow cytometry and FACS}

In order to isolate cell subpopulations, SVFs were stained for $1 \mathrm{~h}$ on ice using primary antibodies against CD144 (AHP628Z; AbD Serotec-BioRad), CD45-FITC (MCA832F; BioRad), CD146-AF647 (MCA2141A647; AbD Serotec-BioRad), and CD34-PE (4H11[APG]; ImmunoTools) followed by incubation for $30 \mathrm{~min}$ on ice with AF405-conjugated secondary antibody (ab175654; AbCam). Isotype controls used (raised in mouse) were IgG1FITC (MCA928F; AbD serotec-BioRad), IgG1-AF647 (MCA928A647, AbD serotec-BioRad), and IgG1-PE (21275514; ImmunoTools). Fluorescence minus one (FMO) for CD146 was obtained with a mix of antibodies against CD144, CD45, and CD34, and the mix for CD34 FMO included CD144, CD45, and CD146. DAPI was added to the samples $5 \mathrm{~min}$ before flow analysis and was used to determine cell viability. Cells were sorted on a BD FACSAria Fusion (BD Biosciences, San Jose, CA, USA).

In order to analyze the immunophenotype of cultured cells, non-sorted, $\mathrm{CD} 34^{+}$, and $\mathrm{CD} 146^{+}$cells were harvested using accutase (SLBN3817V; Sigma). The staining protocol was similar to that already described for FACS and included primary antibodies to CD29 (303001; BioLegend), CD44 (MCA1082GA; AbD serotec-BioRad), CD90 (554895; BD Biosciences), and CD105 (MCA1557T; AbD serotec-BioRad), as well as the isotype controls mouse IgG1א (400101; BioLegend) and mouse IgG1 (MAB002: R\&D Systems). Secondary antibody was AF488-conjugated (A11029; Invitrogen-Thermo Fisher Scientific), and Sytox blue (S34857; Thermo Fisher Scientific) was used to determine cell viability. Samples were run on a BD LSR Fortessa
(BD Biosciences) and data were analyzed using BD FACSDiva software version 8.0.1 or Flowjo (LLC, Ashland, OR, USA).

\section{RNA extraction and gene expression analyses}

Cultured cells or freshly collected SVFs were harvested in Trizol (15596-026; Invitrogen-Thermo Fisher Scientific) and the concentration of total RNA was measured using Nanodrop. RNA was reverse transcribed with SuperScript III (18080-044; Invitrogen-Thermo Fisher Scientific) and qPCR was performed using the SensiFAST SYBR Lo-ROX kit (BIO-94020; Bioline, London, UK) in a Stratagene machine. Primers were either designed by us for CD144 (5'-TCTGCAGGACATCAATGACAAC-3' and $5^{\prime}$-CTTCAG GCACGGCAAATACG-3') and $18 \mathrm{~S}$ (5'-GCTGGCACCAGACTTG-3' and 5'-GGGGAATC AGGGTTCG-3') or were based on previously reported sequences [24]. Data were analyzed with MxPro software and normalized to $18 \mathrm{~S}$.

\section{Cell differentiation}

Non-sorted, CD34 ${ }^{+}$, and CD $146^{+}$cultured cells were differentiated into adipocytes, chondrocytes, and osteocytes. Briefly, for adipocyte differentiation, cells were grown until $70 \%$ confluency $[25,26]$ and induced in the presence of dexamethasone (1 $\mu \mathrm{m}, \mathrm{D} 4902$; Sigma Aldrich), 3-isobutyl1-methylxanthine (0.5 mM, 15879; Sigma Aldrich), insulin $(10 \mu \mathrm{g} / \mathrm{ml}, 19278$; Sigma Aldrich), indomethacin $(100 \mu \mathrm{M}$, I8280; Sigma Aldrich), rabbit serum (7\%), and fetal bovine serum (3\%). Cells were differentiated for 5 days with medium being changed twice. Chondrogenesis and osteogenesis were performed using StemPro differentiation kits following the manufacturer's instructions for 18 days (A10071-01 and A10072-01, respectively; Life technologies-Thermo Fisher Scientific). Adipocytes were stained with Oil red O (O0625; Sigma-Aldrich) as described before [27]. In brief, cells were first fixed in paraformaldehyde (PFA; 4\%) for 3 min, rinsed with $60 \%$ isopropanol, and stained with Oil red $\mathrm{O}(0.1 \%)$ for $10 \mathrm{~min}$ and then rinsed with isopropanol again and washed with water. Chondrocyte pellets were embedded in Histogel (HG-4000; Invitrogen-Thermo Fisher Scientific) and paraffined, and then cut at $8 \mu \mathrm{m}$ thickness. Slides were dewaxed and rehydrated. Alcian blue solution (1\%) was added to the slide and incubated overnight. After washing, Neutral red (1\%) solution was added and absolute ethanol and xylene were used to dehydrate the slides that were mounted with pertex. For osteocyte staining, cells were washed with PBS, fixed with PFA (4\%) for $15 \mathrm{~min}$, and stored in PBS at $4{ }^{\circ} \mathrm{C}$. After washing the cells with water, Alizarin Red S (A5533; Sigma-Aldrich) was added. The plate was incubated at room temperature in the dark for $10 \mathrm{~min}$ and washed with water. Alkaline phosphatase activity was 
determined in differentiated cells using a commercial kit (86R-1KT; Sigma-Aldrich) according to the manufacturer's specifications. Briefly, cells were washed twice with PBS, then fixed with PFA-citrate acetate buffer for $30 \mathrm{sec}$ and washed again with PBS, to which the alkaline-dye mixture was added for the reaction to proceed with wells protected from light, and then washed with water. Negative controls were produced with nondifferentiated cells in culture and micrographs were taken on an Axiovert 25 inverted microscope (Zeiss, Oberkochen, Germany).

\section{Angiogenesis assays}

The chorioallantoic membrane (CAM) assay was used to assess in vivo angiogenic potential of non-sorted cells and sorted $\mathrm{CD}_{3}{ }^{+}$and $\mathrm{CD} 146^{+}$cells. Fertilized eggs (NovoGen, Le Foeil, France) were cleaned with ethanol (70\%) and incubated at $37{ }^{\circ} \mathrm{C}$ in $60 \%$ humidity. Three days later, $2 \mathrm{ml}$ of albumen was aspirated from the egg with a syringe and a window was created which was covered again and returned to incubation. On day 8 of incubation, polypropylene rings were placed on the CAM and cells $(75,000$ cells) or PBS were placed in the center of the ring, as described previously [28, 29]. The eggs were incubated for a further 2 days, when images from each ring were taken using the Zeiss AXIO ZoomV16 microscope at $40 \times$. Binary images were generated by Image $\mathrm{J}$ and angiogenesis was quantified using AngioSys2.0 software (Cellworks, Buckingham, UK).

For in vitro angiogenesis experiments, $\mathrm{CD} 146^{+}$and endothelial cells were stained with PKH26 $(20 \mu \mathrm{M})$ and PKH67 $(20 \mu \mathrm{M})$ (both from Sigma-Aldrich), producing red and green fluorescence signals, respectively. Once labeled, cells were resuspended in EGM-2 medium and seeded on ibiTreat $\mu$-Slides (IB-81506; Thistle Scientific) coated with matrigel. Pictures were taken using a Zeiss Live Cell Observer/Deconvolution system.

\section{Statistical analysis}

Results are shown as mean \pm standard error of the mean, and were analyzed by one-way or two-way ANOVA followed by comparison among means with Tukey test using GraphPad Prim 6.0 software (GraphPad Software, La Jolla, CA, USA). Significance was set at $p<0.05$.

\section{Results}

\section{CD146 and CD34 mark perivascular cells and colocalize} with MSC markers in equine tissues

We began by testing the cross-reactivity of cell surface marker antibodies in equine tissues. In human tissues, CD146 (MCAM, Muc18) has been commonly used to identify pericytes surrounding small blood vessels $[16,30]$. Similarly, in equine tissues including adipose, testis, and skeletal muscle, CD146 had an abluminal location to the endothelial marker CD144 (VE-cadherin); importantly, CD144 was not present in CD146 ${ }^{+}$cells, indicating that these corresponded to pericytes (Fig. $1 \mathrm{a}-\mathrm{c}$ ). Other antigens known to be expressed by pericytes, namely NG2 (CSPG4), $\alpha$ SMA (ACTA1), and CD146, colocalized in the tissues analyzed (Fig. 1d, e). Moreover, the MSC markers CD29 and CD44 were also detected perivascularly, costaining with $\mathrm{NG} 2$ and CD146, respectively (Fig. 1f, g). Adventitial cells were identified by CD34 ${ }^{+}$ staining in the outer layer of larger blood vessels, which followed a pattern similar to that of the MSC marker CD44 (Fig. 1h, i). Overall, these results showed that pericytes and adventitial cells colocalize perivascularly with MSC markers in horse tissues, consistent with the notion in the human that perivascular cells give rise to MSCs in culture [15, 17].

\section{Isolation of $\mathrm{CD} 146^{+}$and $\mathrm{CD} 34^{+}$cells from equine adipose tissue}

There are no reports on the isolation of perivascular cells in the horse. Extraction of the stromal vascular fraction (SVF) from adipose tissue by standard collagenase digestion resulted in an adipocyte-free preparation (around 300,000 cells/g tissue) containing different cell types including many erythrocytes. Thus, an essential step in the preparation of samples for FACS was incubation with red blood cell lysis buffer, because high numbers of erythrocytes could interfere with antibody staining, flow analysis, and cell sorting. Selection of an antibody panel for FACS was based on IHC validation (Fig. 1) as well as optimization by flow cytometry using equine adipose, testes, and peripheral blood mononuclear cell samples (data not shown). FACS of CD34 $4^{+}$ and $\mathrm{CD} 146^{+}$subpopulations from adipose SVF extracts sequentially involved: selection of single events (single cells) plotted as forward scatter area vs forward scatter height (FSC-A vs FSC-H; Fig. 2A), visualized in the dotplot of FSC-A vs side scatter area (SSC-A; Fig. 2B); selection of DAPI-negative (live) cells (Fig. 2C); sorting out of $\mathrm{CD}_{144^{+}}$endothelial cells (Fig. 2D); gating of CD45-FITC-negative cells (Fig. 2E); and selection of cells positive for CD34-PE and CD146-AF647 (Fig. 2F) to separately obtain $\mathrm{CD} 45^{-} / \mathrm{CD} 144^{-} / \mathrm{CD} 146^{-} / \mathrm{CD} 34^{+}$ $\left(\mathrm{CD} 34^{+}\right.$cells; $0.6 \pm 0.4 \%$ of total) and $\mathrm{CD} 45^{-} / \mathrm{CD} 144^{-} /$ $\mathrm{CD}^{-} 4^{-} / \mathrm{CD} 146^{+}$cells $\left(\mathrm{CD} 146^{+}\right.$cells; $1.9 \pm 0.7 \%$ of total). Antibody isotypes or AF405-linked secondary antibodies were used as controls (Fig. 2c2, c4, c5). CD34 ${ }^{+}$ and $\mathrm{CD} 146^{+}$cells were then cultured and characterized.

\section{$\mathrm{CD} 146^{+}$and $\mathrm{CD} 34^{+}$cells show similar growth in culture}

Non-sorted cells (conventional MSCs) and sorted CD $34^{+}$ and $\mathrm{CD}_{146^{+}}$cells showed similar morphology in culture (Fig. 3a). Cells were passaged and seeded at a density of 5000 cells $/ \mathrm{cm}^{2}$, producing similar doubling times between 

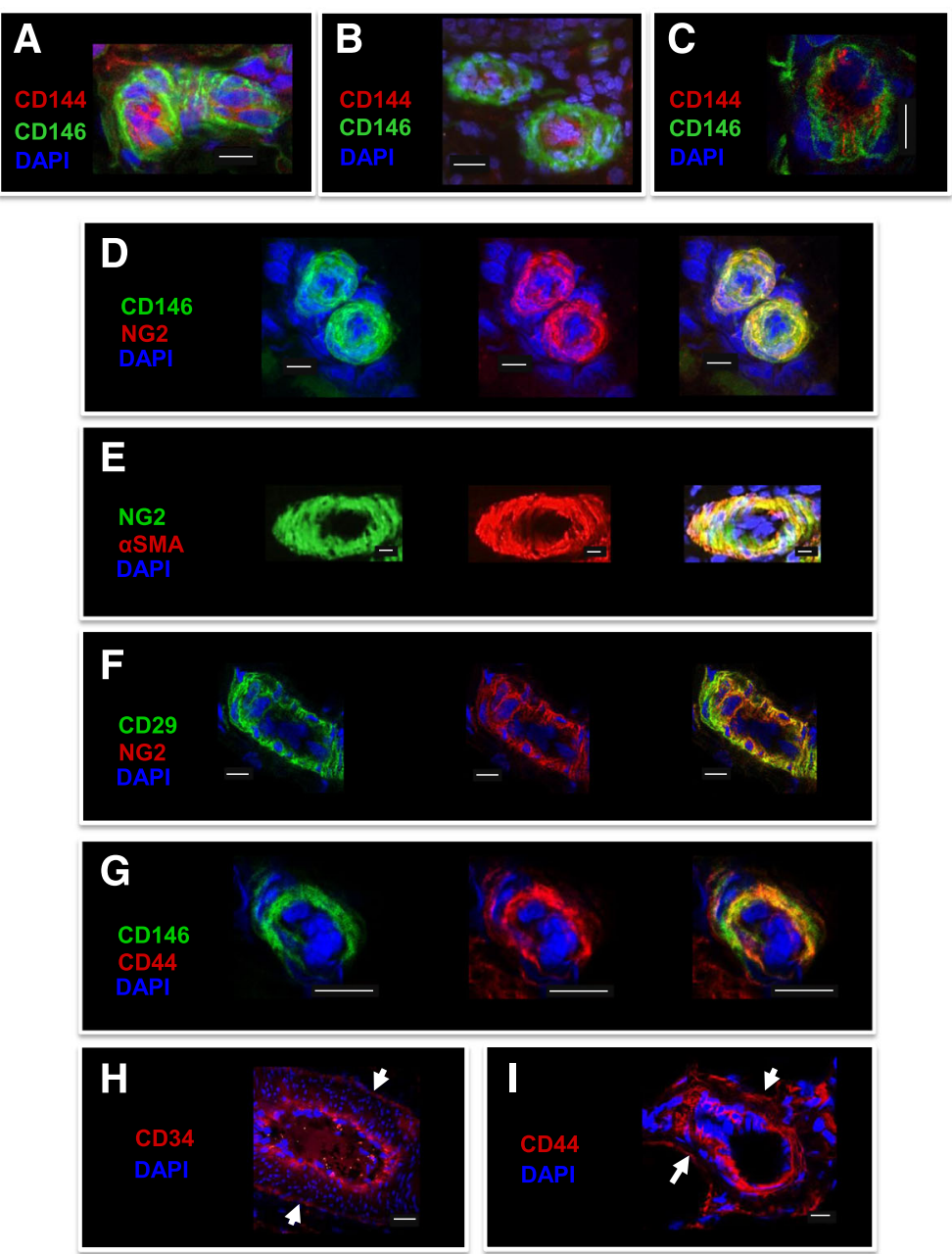

Fig. 1 Immunohistochemistry of pericyte, adventitial cell, and MSC markers in equine tissues. a-c Pericytes stained for CD146 surrounding CD144 + endothelial cells in adipose tissue (a), testis (b), and skeletal muscle (c). d, e Dual staining (right panel) with the pericyte markers NG2 and CD146 (d) or NG2 and aSMA (e) in adipose tissue and testis, respectively. $\mathbf{f}, \mathbf{g}$ Colocalization (right panel) of the MSC and pericyte markers CD29 and NG2 (f) and CD44 and CD146 (g) in adipose tissue, respectively. $\mathbf{d}-\mathbf{g}$ Individual staining is shown (left and middle panels). $\mathbf{h}$, i Immunodetection of the adventitial cell marker CD34 (h) and the MSC marker CD44 (i) in the outer layer of blood vessels (arrows) in adipose tissue. 4',6-Diamidino2-phenylindole (DAPI) was used to stain cell nuclei. White scale bars $=10 \mu \mathrm{m}$

passages 2 and 6 (Fig. 3b). CFU-F (Fig. 3c) were quantified by seeding cells at low densities $\left(1,5\right.$, and 25 cells $\left./ \mathrm{cm}^{2}\right)$ and staining colonies with crystal violet 12 days later. In our hands, a seeding density of 5 cells $/ \mathrm{cm}^{2}$ was optimal and was used for CFU-F quantification, with no significant differences being found between the three cell types (Fig. 3d).

\section{Expression of cell surface markers by $\mathrm{CD} 146^{+}$and $\mathrm{CD} 34^{+}$ cells in culture}

Based on $\mathrm{qPCR}$ analyses, $\mathrm{CD} 146^{+}$cells expressed the highest levels of CD146 throughout culture, around 3fold higher than non-sorted cells $(p<0.05$; Fig. 4a), showing that the isolation process was effective. CD146
mRNA was detected in some $\mathrm{CD} 4^{+}$cell samples but at markedly reduced levels compared with $\mathrm{CD}_{146^{+}}$cells (27-fold lower in average), which may correspond to a transitional minority subpopulation between pericyte and adventitial cells as described in humans [30]. Expression of CD34 was variable and substantial only in $\mathrm{CD}_{3} 4^{+}$cells at passages $1 / 2$ and then decreased (Fig. 4b; $p<0.05$ ), as is known to occur in cells in culture $[31,32]$. CD34 was absent in the CD146 ${ }^{+}$cells, except for very low levels in one of the samples. CD144 was detected in all non-sorted samples (Fig. 4c) at lower values than in the initial SVF crude extract (data not shown). CD144 was expressed at much lower levels (around 13-fold) in $\mathrm{CD}^{+} 4^{+}$cells and was occasionally 

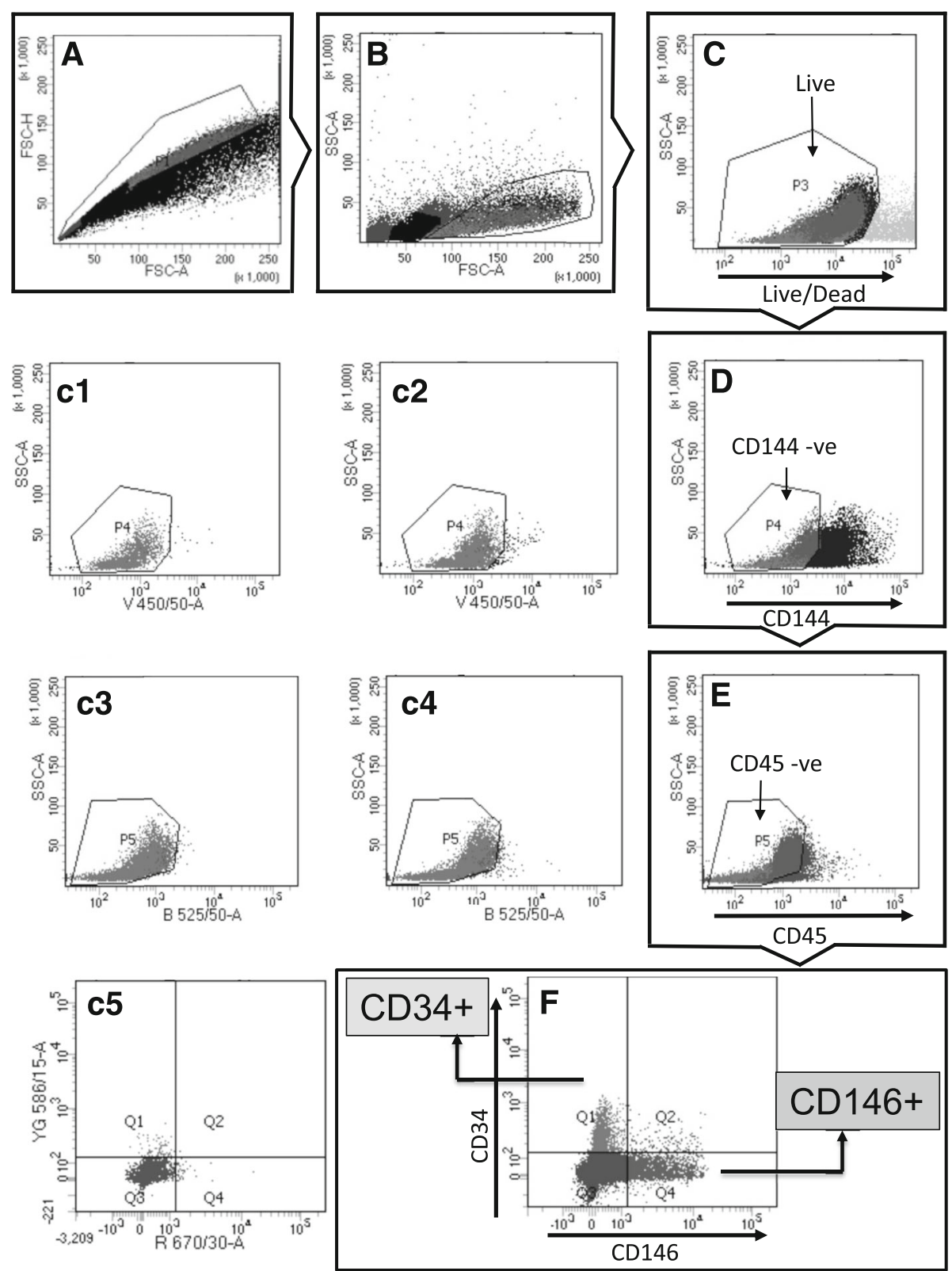

Fig. 2 Isolation of $\mathrm{CD}_{4} 4^{+}$and $\mathrm{CD} 146^{+}$cells from adipose SVF. $\mathbf{a}, \mathbf{b}$ Events displayed as FSC-A vS FSC-H to select singlets (a) were further gated to exclude cell fragments or noncellular material (b). c-e Sequence of plots showing selection of DAPI-negative, CD144(AF405)-negative, and CD45(FITC)-negative cells to obtain live, endothelial-negative, and hematopoietic-negative fractions, respectively. (c1-c4) Dot-plots for CD144 $(c 1, c 2)$ and $C D 45(c 3, c 4)$ controls showing unstained cells $(c 1, c 3)$ and secondary antibody conjugated to AF405 (c2) or isotype conjugated to FITC (c4). f Double-plot displaying $\mathrm{CD}_{3} 4^{+}(\mathrm{Q} 1)$ and $\mathrm{CD} 146^{+}(\mathrm{Q} 4)$ cell subpopulations and respective isotype controls (c5) conjugated to PE and AF647, respectively. Filters used: 450/50 for AF405, 525/50 for FITC, 586/15 for PE, and 670/30 for AF647. FSC-A forward scatter area, FSC-H forward scatter height, SSC-A side scatter area

detected, at negligible levels, in $\mathrm{CD}_{146^{+}}$cells. CD45 was not detected except in one of the non-sorted preparations at passages $1 / 2$ (data not shown), an observation that we have occasionally made in other non-sorted samples and which has also been reported by others [33].
$\mathrm{CD} 146^{+}$and $\mathrm{CD} 34^{+}$cells express MSC markers

qPCR analysis demonstrated that MSC markers were present in both $\mathrm{CD}_{3} 4^{+}$and $\mathrm{CD} 146^{+}$cells in culture (Fig. 5). Indeed, CD73, CD90, and CD105 mRNA was detected in both cell types as well as in non-sorted cells up to at least passage 4 (Fig. 5a). Expression of these 


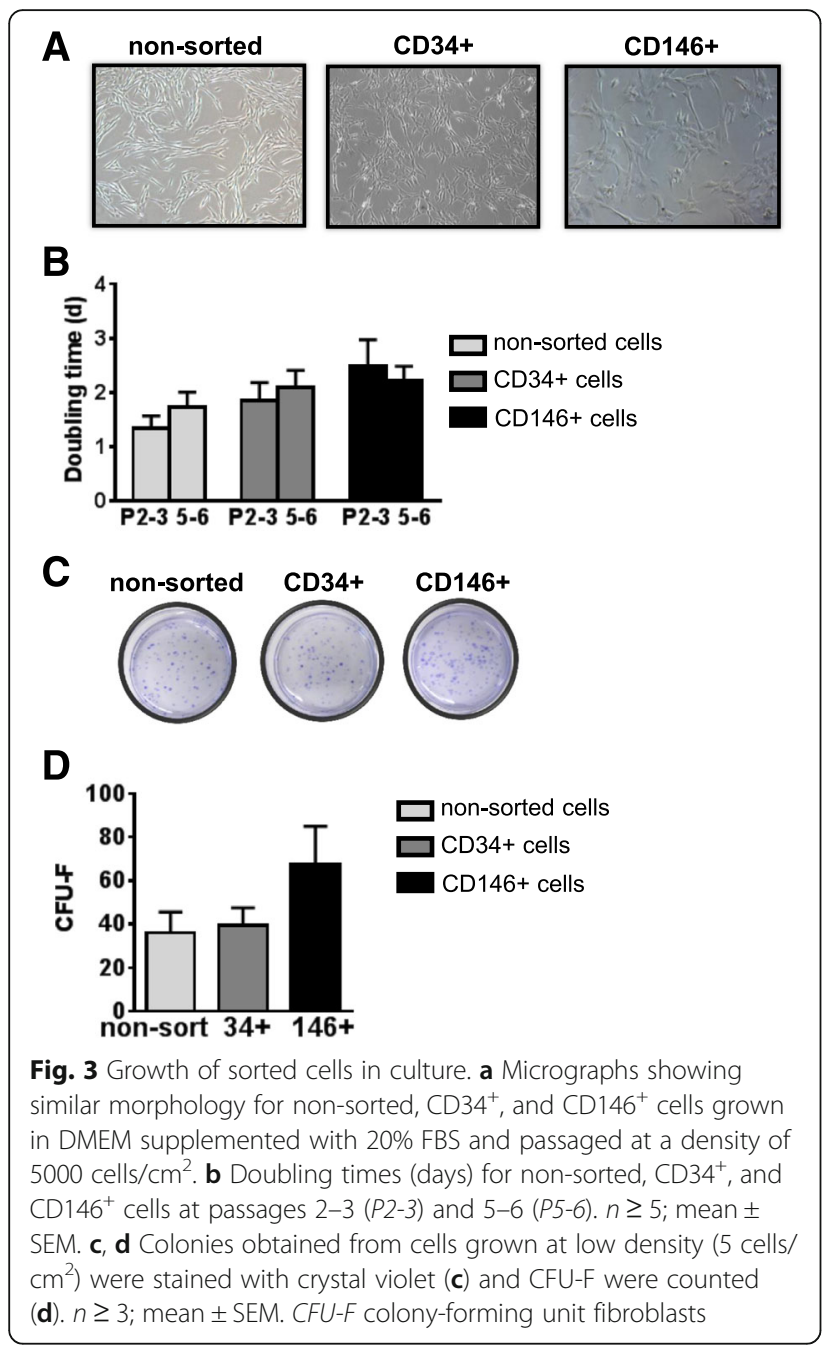

genes was lower in $\mathrm{CD} 146^{+}$cells than in non-sorted cells at all passages $(p<0.05)$, except for CD90 at passages 3/4. Moreover, apart from higher $(p<0.05)$ expression of CD105 in $\mathrm{CD}^{+} 4^{+}$cells than $\mathrm{CD} 146^{+}$ cells at passages $1 / 2$, there were no differences in expression of the three MSC markers between $\mathrm{CD} 34^{+}$ and the other two cell types.

In agreement with the $\mathrm{qPCR}$ results, all MSC markers tested by flow cytometry (CD29, CD44, CD90, and CD105) were present in $\mathrm{CD}_{4} 4^{+}$and $\mathrm{CD} 146^{+}$cells (Fig. 5b). Importantly, for all markers the antibody-positive cells produced distinct peaks from the isotype controls, indicating that the great majority of the cells in the samples expressed the respective maker. However, differences were observed in mean fluorescence intensity (MFI), suggesting differences in antigen level between cell types (Fig. 5c). In general, MFI values for MSC markers were lower in $\mathrm{CD}_{146^{+}}$cells than in non-sorted and $\mathrm{CD}_{3} 4^{+}$cells, although these differences were not significant in the case of CD44.

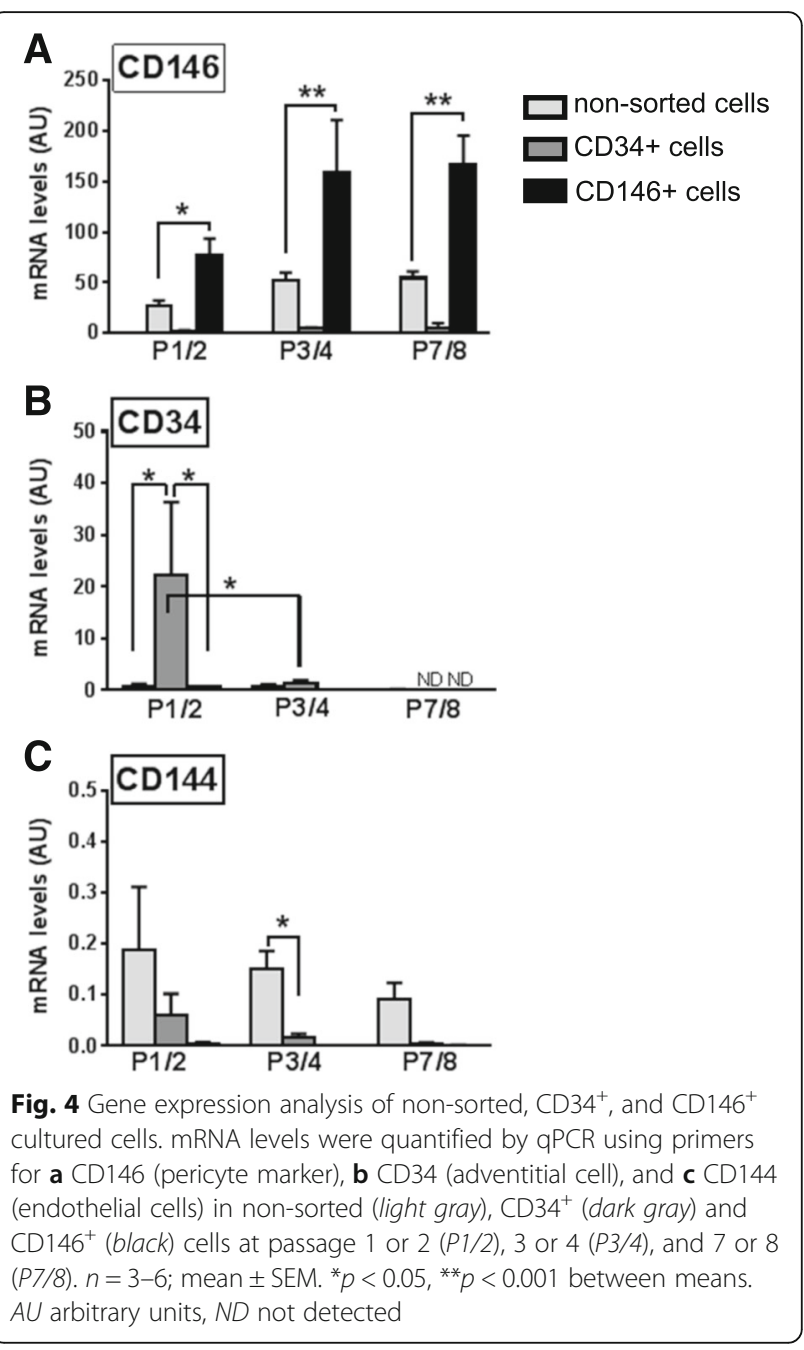

\section{$\mathrm{CD}_{146}{ }^{+}$and $\mathrm{CD} 34^{+}$cells are multipotent}

Similar to non-sorted cells, both $\mathrm{CD}^{+} 4^{+}$and $\mathrm{CD} 146^{+}$ populations were capable of trilineage differentiation (Fig. 6). Upon culture in adipogenic media, all three cell types became round in morphology and accumulated lipid vesicles, as shown by Oil red $\mathrm{O}$ staining in the cytoplasm (Fig. 6a). Chondrogenesis and osteogenesis were also observed in the three cell types, with Alcian blue coloring glycosaminoglycanss in chondrocyte pellets counterstained with Neutral red (Fig. 6b). Osteocytes displayed staining of calcium deposits by Alizarin Red and were also alkaline phosphatase-positive (Fig. 6c). These results indeed show that multipotency is maintained by $\mathrm{CD} 146^{+}$and CD34 ${ }^{+}$cells in culture.

\section{Equine $\mathrm{CD} 146^{+}$cells promote angiogenesis}

Angiogenesis is crucial in tissue regeneration and can be reportedly stimulated by MSCs [34]. For this reason, we determined the relative expression of the angiogenic factors VEGFA and ANGPT1 by CD $34^{+}, \mathrm{CD} 146^{+}$, and non-sorted cells, as well as the capacity of each cell type 

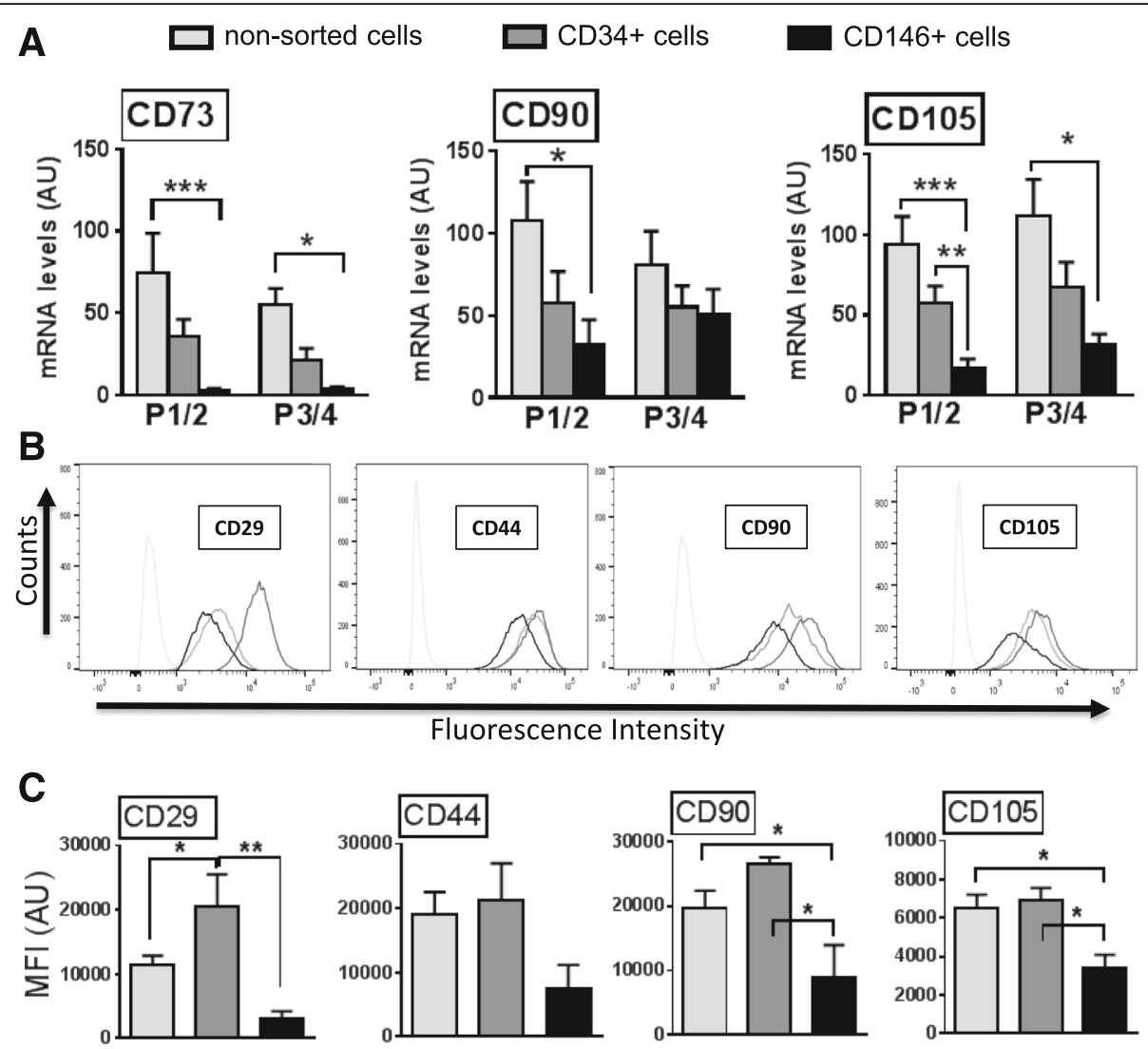

Fig. 5 Isolated CD146 and CD34+ cells express MSC markers. qPCR measurements (a) of CD73 (left), CD90 (middle), and CD105 (right) of non-sorted (light gray), CD34 (dark gray), and CD146 (black) cells analyzed at passage 1 or 2 (P1/2) and 3 or 4 (P3/4). Flow cytometry histograms (b) displaying fluorescence intensity (AF488 conjugated to the secondary antibody) vs event counts (corresponding to number of cells) and showing displacement of non-sorted (light gray), CD34 (dark gray), and CD146 (black) cultured cells stained with antibodies CD29, CD44, CD90, and CD105 in comparison with the isotype controls (very light gray, on left). Data were obtained from P3 to P5. c Quantification of the flow signals depicted in bar graphs as mean fluorescence intensity (MFI) of non-sorted (light gray), CD34 ${ }^{+}$(dark gray), and CD146 ${ }^{+}$(black). $n \geq 3$; mean \pm SEM. ${ }^{*} p<0.05,{ }^{* *} p<0.001$, ${ }^{* * *} p<0.0001$ between means. AU arbitrary units

to promote angiogenesis in vivo (Fig. 7). The three cell types expressed VEGFA and ANGPT1, but levels of the two genes were significantly higher in $\mathrm{CD} 146^{+}$cells than in non-sorted or CD34 $4^{+}$cells (Fig. 7a). We then tested angiogenic activity using the chick embryo chorioallantoic membrane (CAM) assay, a method that has been extensively used for angiogenic assays in tumor [28] and stem cell [34-36] studies. The CAM is easily accessible and allows quantification of angiogenesis in a large number of samples simultaneously. In agreement with qPCR data, we found that $\mathrm{CD} 146^{+}$cells distinctly promoted angiogenesis in vivo, as shown by significant increases (relative to PBS control) in blood vessel branching and total vessel length in membranes exposed to CD146 ${ }^{+}$cells compared with non-sorted and $\mathrm{CD} 34^{+}$cells (Fig. 7b, c). In addition, coculture experiments (Fig. $7 \mathrm{~d}$ ) demonstrated the association between $\mathrm{CD}_{146^{+}}$cells and endothelial cells on matrigel, suggestive of network stabilization by $\mathrm{CD} 146^{+}$as occurs naturally in tissues, and indicating that these cells maintained their native properties in culture.

\section{Discussion}

Despite the enormous interest in the use of MSC therapies in both humans and horses, and the impressive amount of literature devoted to this topic over the last decade, cell heterogeneity in clinical MSC preparations has significantly hindered the full exploitation of these therapies. The identification of two distinct perivascular cell subpopulations, based on the expression of CD146 and CD34, as precursors of MSCs in human tissues [15, 17] provided a significant step forward toward new therapies with increased clinical efficacy. However, studies using defined cell subpopulations (as opposed to traditional heterogeneous MSC preparations) in the horse are lacking, largely a consequence of the difficulty in translating cell isolation procedures developed for human cells to other species and the limited availability of species-specific antibodies. Here, we have successfully established for the first time a methodology to isolate perivascular CD34 ${ }^{+} / \mathrm{CD} 146^{-} / \mathrm{CD} 45^{-} / \mathrm{CD} 144^{-}$and $\mathrm{CD} 146^{+} / \mathrm{CD}^{-} 4^{-} / \mathrm{CD} 45^{-} /$ $\mathrm{CD} 144^{-}$populations in the horse. We report the successful 


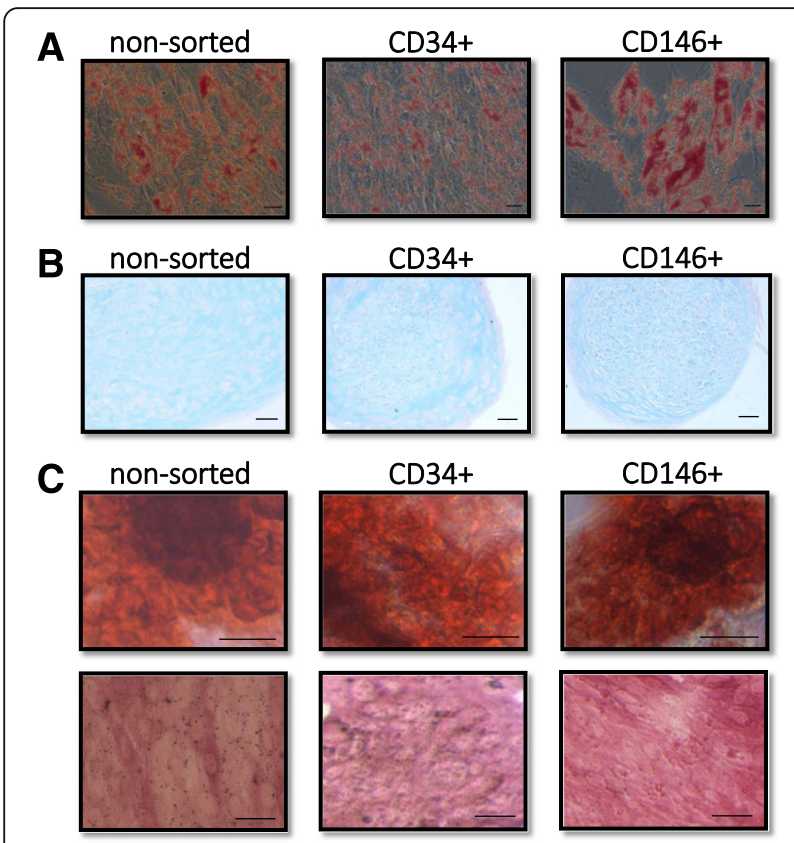

Fig. 6 Isolated $\mathrm{CD}_{146^{+}}$and $\mathrm{CD} 34^{+}$cells are multipotent. Micrographs showing differentiation of non-sorted (left), CD34 ${ }^{+}$(middle), and CD146 ${ }^{+}$ cells (right) into adipocytes (a), chondrocytes (b), and osteocytes (c). a Adipocytes were stained with Oil red $\mathrm{O}$, showing the lipid content in the cells (red). $\mathbf{b}$ Histological sections $(8 \mu \mathrm{m})$ of differentiated chondrocyte pellets were stained with Alcian blue to detect glycosaminoglycans and counterstained with Neutral red. c Alizarin Red $\mathbf{S}$ and the alkaline phosphatase activity assay were used to stain osteocytes differentiated for 19 days, resulting in staining of calcium deposits (red, upper row and purple, lower row, respectively). Black scale bars $=50 \mu \mathrm{m}$

expansion of these cells in culture as well as the characterization of their properties relative to nonsorted MSC populations.

The observation that CD146 and CD34 have a perivascular location in different equine tissues which coincides with that of established MSC markers is consistent with the notion that pericytes and adventitial cells are native MSC precursors [15, 17, 37], thus extending previous observations in humans to the horse. Our finding that CD146 is robustly maintained by sorted CD146 ${ }^{+}$cells in culture is in agreement with two previous PCR [38] and flow cytometry [39] studies reporting the presence of CD146 in equine MSCs. In contrast, expression of CD34 in $\mathrm{CD} 34^{+}$cells was variable and quickly decreased upon culture, as reported for other cells [31, 32]. This may account in part for the fact that CD34 has only occasionally been detected by PCR in equine MSCs [38, 40], and for the difficulty so far in identifying CD34 antibodies cross-reacting with equine cells. Indeed, in the present study only one of the five CD34 antibodies tested could be used successfully with equine samples.

$\mathrm{CD} 34^{+}$and $\mathrm{CD} 146^{+}$populations displayed typical MSC properties, namely adherence to uncoated tissue-culture dishes, inclusion of CFU-F, and trilineage differentiation, in addition to expressing classical MSC markers both in tissues and during culture. Importantly, flow cytometry analyses indicated that these markers were present in almost all cells within the two sorted subpopulations, although corresponding MFI and mRNA levels were in general lower in $\mathrm{CD} 146^{+}$cells than in the other cell types analyzed. This could be attributed to loss of cell surface marker expression during the time in culture. Another possibility is that cell contaminants such as hematopoietic and/or endothelial cells, which can also express MSC markers [41-45], contributed to the relatively higher expression of these markers, particularly in non-sorted cell cultures. Consistent with this assumption, the single non-sorted cell sample with detectable CD45 mRNA also displayed the highest values for CD90 and CD105 and, in addition, the endothelial marker CD144 was also expressed by non-sorted cells in culture. Regardless of the reasons for these differences, the results indicate that $\mathrm{CD} 146^{+}$cells are distinct from non-sorted and CD $34^{+}$cells in equine adipose tissue.

Angiogenesis has a critical contribution to tissue regeneration by allowing supply of oxygen, nutrients, and immune cells and clearing of metabolic products [46, 47]. Because of their anatomical location, pericytes and adventitial cells are uniquely positioned to regulate angiogenesis both by direct contact with endothelial cells and by paracrine production of angiogenic factors [23, 48, 49]. Using the CAM model, we showed that, under our experimental conditions, $\mathrm{CD} 146^{+}$cells, but not non-sorted or $\mathrm{CD} 34^{+}$ cells, were able to promote angiogenesis in vivo, presumably due in part to their higher expression of the angiogenic factors VEGFA and ANGPT1. In the horse, two publications $[50,51]$ reported on the angiogenic activity of MSCs, specifically on the stimulation of vascular network formation by peripheral blood-derived MSCs in culture [50] and the proangiogenic effects of adipose MSC-derived membrane vesicles in rat aortic rings and scratch assays [51]. However, in those reports the effects of MSCs in vivo were not determined and, in addition, the specific subpopulations involved in the responses observed in vitro were not identified. Limited studies in humans have gone further to determine the angiogenic effects of isolated $\mathrm{CD} 146^{+}$or $\mathrm{CD} 34^{+}$populations in vivo using animal models. In particular, human $\mathrm{CD}_{146^{+}}$cells were shown to promote angiogenesis after transplantation into SCID/mdx mouse muscle [52] and in SCID mouse ischemic myocardium [22]. Moreover, improvement of angiogenesis in ischemia was reported in response to $\mathrm{CD} 34^{+} / \mathrm{CD} 31^{-}$cells obtained from human saphenous vena; however, other cell types in these preparations could have contributed to the effects observed [53]. Importantly, the effects of $\mathrm{CD} 146^{+}$and $\mathrm{CD} 34^{+}$on 

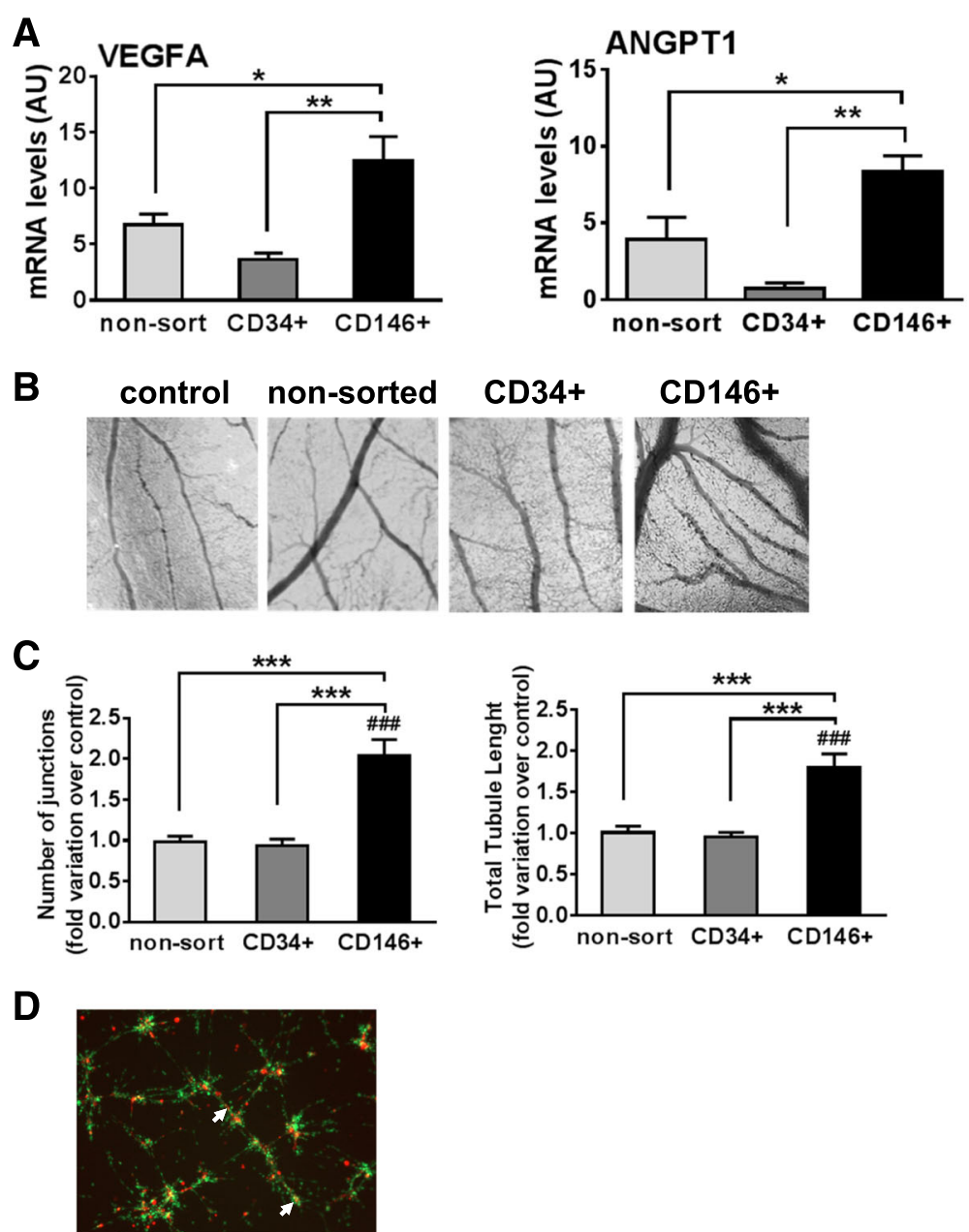

Fig. $7 \mathrm{CD} 146^{+}$cells are angiogenic. a Transcript levels of angiogenic factors VEGFA and ANGPT1 in non-sorted (light gray), CD34 (dark gray), and CD146 ${ }^{+}$(black) cells, quantified by qPCR. b CAM micrographs obtained at 40x magnification showing formation of blood vessel plexus at 2 days following incubation with PBS control (left), non-sorted (middle left), CD34 (middle right), and CD146 (right) cells. c Quantification of the CAM blood vessel plexus using AngioSys2.1 software as fold variation in blood vessel branching (left) and blood vessel total length (right) over PBStreated controls. Non-sorted (light gray), CD34 (dark gray), and CD146 (black) cells. d Micrographs of in vitro cocultures of equine endothelial (green, labeled with PKH26; $20 \mu \mathrm{M}$ ) and $\mathrm{CD}_{146}{ }^{+}$(red, labeled with PKH67; $20 \mu \mathrm{M}$ ) cells seeded on matrigel, showing close apposition of CD146 ${ }^{+}$ cells to the endothelial network (white arrows). $n \geq 3$ for $\mathrm{qPCR}, n \geq 12$ for CAM experiments; mean \pm SEM. ${ }^{*} p<0.05,{ }^{* *} p<0.001,{ }^{* * *} p<0.0001$, differences between different cell types; \#\#\#p <0.0001, difference between CAM incubated with CD146 ${ }^{+}$cells and with PBS (control). AU arbitrary units

angiogenesis had so far not been directly compared in any species. Our results using the equine model show CD146 ${ }^{+}$ cells to be distinctly angiogenic compared with $\mathrm{CD} 34^{+}$ cells and suggest that the former subpopulation is a primary contributor to the proangiogenic properties attributed to MSCs.

\section{Conclusions}

Here we report the isolation and characterization of $\mathrm{CD}_{146^{+}}$and $\mathrm{CD}_{3} 4^{+}$cell subpopulations from equine adipose tissue. The results showed that although both subpopulations display a MSC-like phenotype, CD146 ${ }^{+}$ cells are distinctly angiogenic in vivo, indicating CD146 cells maintain a pericyte phenotype during culture. Based on these results, the marker panels currently used to define MSC populations with regenerative potential may have to be reassessed, because high levels of CD146, but not the classical MSC markers, were associated with angiogenic capacity in this study. Our results also underscore the potential advantages of using defined cell populations over standard heterogeneous MSC preparations for therapy. Angiogenesis is indeed a major component of tissue repair and the use of CD146 ${ }^{+}$ cell subpopulations may be particularly beneficial in the 
future to improve the efficacy of regenerative treatments both in horses and humans.

\section{Abbreviations}

CAM: Chorioallantoic membrane; CFU-F: Colony-forming unit fibroblasts; DAPI: 4',6-Diamidino-2-phenylindole; FACS: Fluorescence-activated cell sorting; FMO: Fluorescence minus one; FSC: Forward scatter; MFI: Mean fluorescence intensity; MSC: Mesenchymal stem/stromal cells; PFA: Paraformaldehyde; SSC: Side scatter; SVF: Stromal vascular fraction

\section{Acknowledgements}

The authors would like to thank Dr John Keen and Dr Ruth Morgan for providing tissue samples and Mr Neil Maclntyre and the staff at the Pathology Services in the Royal (Dick) School of Veterinary Studies for their help.

\section{Funding}

This work was supported by the Horserace Betting Levy Board (Prj768) and an Institute Strategic Programme Grant from The Biotechnology and Biological Sciences Research Council.

\section{Availability of data and materials}

Not applicable.

\section{Authors' contributions}

CLE, TAS, SPM, JJP, TM, and LD performed all experiments and collected data. CLE and FXD analyzed the data. CLE, BP, and FXD conceived and design the project and wrote the manuscript. All authors approved the final manuscript.

\section{Author's information}

Not applicable.

\section{Competing interests}

$\mathrm{BP}$ is coinventor of human perivascular stem cell-related patents filed from University of California, Los Angeles. All authors declare they have no competing interests.

\section{Consent for publication}

All authors have approved the manuscript and agree to submit to this journal.

\section{Ethics approval}

All animal procedures were carried out according to the UK Home Office Animals (Scientific Procedures) Act 1986 (60/4207) with approval by the Ethical Review Committee, University of Edinburgh.

\section{Publisher's Note}

Springer Nature remains neutral with regard to jurisdictional claims in published maps and institutional affiliations.

\section{Author details}

${ }^{1}$ The Roslin Institute, University of Edinburgh, Edinburgh, UK. ${ }^{2}$ Centre for Regenerative Medicine, University of Edinburgh, Edinburgh, UK. ${ }^{3}$ Orthopaedic Hospital Research Centre, University of California, Los Angeles, CA, USA. ${ }^{4}$ The Roslin Institute, University of Edinburgh, Easter Bush, Midlothian EH25 9RG, UK.

Received: 31 January 2017 Revised: 23 February 2017 Accepted: 28 February 2017 Published online: 18 April 2017

\section{References}

1. Donadeu FX, Esteves CL. Stem cells and equine health. Euro Stem Cells. 2015. http://www.eurostemcell.org/stem-cells-and-equine-health. Accessed 27 Feb 2015.

2. Smith RKW, Garvican ER, Fortier LA. The current 'state of play' of regenerative medicine in horses: what the horse can tell the human. Regen Med. 2014;9:673-85.

3. Burk J, Badylak SF, Kelly J, et al. Equine cellular therapy—from stall to bench to bedside? Cytom Part A. 2013;83A:103-13.

4. Ranera B, Barry F. A horse of a different color. Cytom Part A. 2014;85:658-9.

5. Brehm W, Burk J, Delling U. Application of stem cells for the treatment of joint disease in horses. Methods Mol Biol. 2014;1213:215-28.
6. Donadeu FX, Esteves CL. Prospects and challenges of induced pluripotent stem cells in equine health. Front Vet Sci. 2015;2:59.

7. Breton A, Sharma R, Diaz AC, et al. Derivation and characterization of induced pluripotent stem cells from equine fibroblasts. Stem Cells Dev. 2013;22:611-21.

8. Sharma R, Livesey MR, Wyllie DJ, et al. Generation of functional neurons from feeder-free, keratinocyte-derived equine induced pluripotent stem cells. Stem Cells Dev. 2014;23:1524-34.

9. Burk J, Ribitsch I, Gittel C, et al. Growth and differentiation characteristics of equine mesenchymal stromal cells derived from different sources. Vet J. 2013;195:98-106.

10. Smith R. Mesenchymal stem cell therapy for equine tendinopathy. Disabil Rehabil. 2008:30:1752-8.

11. Ranera B, Ordovas L, Lyahyai J, et al. Comparative study of equine bone marrow and adipose tissue-derived mesenchymal stromal cells. Equine Vet J. 2012;44:33-42.

12. De Schauwer C, Meyer E, Van de Walle GR, et al. Markers of stemness in equine mesenchymal stem cells: a plea for uniformity. Theriogenology. 2011;75:1431-43.

13. Esteves $C L$, Sharma $R$, Dawson $L$, et al. Expression of putative markers of pluripotency in equine embryonic and adult tissues. Vet J. 2014;202:533-5.

14. Sah RL, Ratcliffe A. Translational Models for Musculoskeletal Tissue Engineering and Regenerative Medicine. Tissue Eng Part B. 2010;16(1):1-3.

15. Crisan $M$, Yap $S$, Casteilla $L$, et al. A perivascular origin for mesenchymal stem cells in multiple human organs. Cell Stem Cell. 2008;3:301-13.

16. Crisan $\mathrm{M}$, Chen C-W, Corselli M, et al. Perivascular multipotent progenitor cells in human organs. Ann New Y Acad Sci. 2009;1176:118-23.

17. Corselli M, Chen CW, Sun B, et al. The tunica adventitia of human arteries and veins as a source of mesenchymal stem cells. Stem Cells Dev. 2012;21:1299-308.

18. Dominici M, Le B, Mueller I, et al. Minimal criteria for defining multipotent mesenchymal stromal cells. The international society for cellular therapy position statement. Cytotherapy. 2006;8:315-7.

19. Bourin P, Bunnell BA, Casteilla L, et al. Stromal cells from the adipose tissuederived stromal vascular fraction and culture expanded adipose tissuederived stromal/stem cells: a joint statement of the International Federation for Adipose Therapeutics and Science (IFATS) and the International Society for Cellular Therapy (ISCT). Cytotherapy. 2013;15:641-8.

20. James AW, Zara JN, Corselli M, et al. An abundant perivascular source of stem cells for bone tissue engineering. Stem Cells Transl Med. 2012;1:673-84.

21. Tawonsawatruk T, West CC, Murray IR, et al. Adipose derived pericytes rescue fractures from a failure of healing —non-union. Sci Rep. 2016;6:22779.

22. Chen CW, Okada M, Proto JD, et al. Human pericytes for ischemic heart repair. Stem Cells. 2013;31:305-16.

23. West CC, Hardy WR, Murray IR, et al. Prospective purification of perivascular presumptive mesenchymal stem cells from human adipose tissue: process optimization and cell population metrics across a large cohort of diverse demographics. Stem Cell Res Ther. 2016;7:47.

24. Ranera B, Lyahyaia J, Romero A, et al. Immunophenotype and gene expression profiles of cell surface markers of mesenchymal stem cells derived from equine bone marrow and adipose tissue. Vet Immunol Immunopathol. 2011:144:147-54.

25. Esteves $\mathrm{CL}$, Kelly $\mathrm{V}$, Breton $\mathrm{A}$, et al. Proinflammatory cytokine induction of 11 beta-hydroxysteroid dehydrogenase type 1 (1 beta-HSD1) in human adipocytes is mediated by MEK, C/EBPbeta, and NF-kappaB/RelA. J Clin Endocrinol Metab. 2014;99:E160-8.

26. Esteves $C L$, Kelly V, Bégay V, et al. Regulation of adipocyte $11 \beta$ hydroxysteroid dehydrogenase type 1 (11ß-HSD1) by CCAAT/EnhancerBinding Protein (C/EBP) $\beta$ isoforms. LIP and LAP. PLoS One. 2012;7, e37953.

27. Esteves $C L$, Kelly V, Bégay V, et al. Stable conditional expression and effect of C/EBP $\beta$-LIP in adipocytes using the pSLIK system. J Mol End. 2013;51:91-8.

28. Ribatti $D$, Nico $B$, Vacca $A$, et al. The gelatin sponge-chorioallantoic membrane assay. Nat Protoc. 2006;1:85-91.

29. Dohle DS, Pasa SD, Gustmann S, et al. Chick ex ovo culture and ex ovo CAM assay: how it really works. J Vis Exp. 2009;33:1620.

30. Zimmerlin L, Donnenberg VS, Pfeifer ME, et al. Stromal vascular progenitors in adult human adipose tissue. Cytom Part A. 2010;77:22-30.

31. Sidney LE, Branch MJ, Dunphy SE et al. Concise review: Evidence for CD34 as a common marker for diverse progenitors. Stem Cells. 2014

32. Braun J, Kurtz A, Barutcu N, et al. Concerted regulation of CD34 and CD105 accompanies mesenchymal stromal cell derivation from human adventitial stromal cell. Stem Cells Dev. 2013;22:815-27. 
33. Radcliffe $\mathrm{CH}$, Flaminio MJ, Fortier LA. Temporal analysis of equine bone marrow aspirate during establishment of putative mesenchymal progenitor cell populations. Stem Cells Dev. 2010;19:269-82.

34. Edwards SS, Zavala G, Prieto CP, et al. Functional analysis reveals angiogenic potential of human mesenchymal stem cells from Wharton's jelly in dermal regeneration. Angiogenesis. 2014;17:851-66.

35. Bronckaers A, Hilkens P, Fanton Y, et al. Angiogenic properties of human dental pulp stem cells. PLoS One. 2013;8, e71104.

36. Wang $\mathrm{C}-\mathrm{H}$, Wang $\mathrm{T}-\mathrm{M}$, Young $\mathrm{T}-\mathrm{H}$, et al. The critical role of ECM proteins within the human MSC niche in endothelial differentiation. Biomaterials. 2013;34:4223-34.

37. Sorrentino A, Ferracin M, Castelli $G$, et al. Isolation and characterization of CD146+ multipotent mesenchymal stromal cells. Exp Hematol. 2008;36:1035-46.

38. Ranera B, Lyahyai J, Romero A, et al. Immunophenotype and gene expression profiles of cell surface markers of mesenchymal stem cells derived from equine bone marrow and adipose tissue. Vet Immunol Immunopathol. 2011;144:147-54.

39. Hoynowski S, Fry M, Gardner B, et al. Characterization and differentiation of equine umbilical cord-derived matrix cells. Biochem Biophys Res Commun. 2007;362:347-53.

40. Lange-Consiglio A, Corradetti B, Bizzaro D, et al. Characterization and potential applications of progenitor-like cells isolated from horse amniotic membrane. J Tissue Eng Regen Med. 2012;6:622-35.

41. Mettouchi A, Meneguzzi G. Distinct roles of $\beta 1$ integrins during angiogenesis. Eur J Cell Biol. 2006;85:243-7.

42. Zhang B. CD73: a novel target for cancer immunotherapy. Cancer Res. 2010 70:6407-11.

43. Narravula S, Lennon PF, Mueller BU, et al. Regulation of endothelial CD73 by adenosine: paracrine pathway for enhanced endothelial barrier function. J Immunol. 2000;165:5262-8.

44. Nassiri F, Cusimano MD, Scheithauer BW, et al. Endoglin (CD105): a review of its role in angiogenesis and tumor diagnosis. progression and therapy. Anticancer Res. 2011;31:2283-90.

45. Hofmeister V, Schrama D, Becker JC. Anti-cancer therapies targeting the tumor stroma. Cancer Immunol Immunother. 2008:57:1-17.

46. Carmeliet P. Angiogenesis in health and disease. Nat Med. 2003;9:653-60.

47. Auerbach $R$, Lewis $R$, Shinners $B$, et al. Angiogenesis assays: a critical overview. Clin Chem. 2003;49:32-40.

48. Murphy MB, Moncivais K, Caplan Al. Mesenchymal stem cells: environmentally responsive therapeutics for regenerative medicine. Exp Mol Med. 2013;45, e54

49. Murray IR, West CC, Hardy WR, et al. Natural history of mesenchymal stem cells, from vessel walls to culture vessels. Cell Mol Life Sci. 2014;71:1353-74.

50. Bussche L, Van de Walle GR. Peripheral blood-derived mesenchymal stroma cells promote angiogenesis via paracrine stimulation of vascular endothelial growth factor secretion in the equine model. Stem Cells Transl Med. 2014;3:1514-25.

51. Pascucci L, Alessandri G, Dall'Aglio C, et al. Membrane vesicles mediate proangiogenic activity of equine adipose-derived mesenchymal stromal cells. Vet J. 2014:202:361-6.

52. Park TS, Gavina M, Chen CW, et al. Placental perivascular cells for human muscle regeneration. Stem Cells Dev. 2011;20:451-63.

53. Campagnolo P, Cesselli D, Al Haj Zen A, et al. Human adult vena saphena contains perivascular progenitor cells endowed with clonogenic and proangiogenic potential. Circulation. 2010;121:1735-45.

\section{Submit your next manuscript to BioMed Central and we will help you at every step:}

- We accept pre-submission inquiries

- Our selector tool helps you to find the most relevant journal

- We provide round the clock customer support

- Convenient online submission

- Thorough peer review

- Inclusion in PubMed and all major indexing services

- Maximum visibility for your research

Submit your manuscript at www.biomedcentral.com/submit 